\title{
CREMONA TRANSFORMATIONS THAT ARE AFFINE AUTOMORPHISMS
}

\author{
HISAO YOSHIHARA
}

\begin{abstract}
We present the condition on which a Cremona transformation induces an automorphism of $\mathbf{A}^{2}$.
\end{abstract}

1. In this paper the ground field is assumed to be an algebraically closed field of characteristic zero. Let $C$ be an irreducible algebraic curve in $\mathbf{P}^{2}$. Then let us call $C$ a curve of type I if $C-\{P\} \cong \mathbf{A}^{1}$ for some point $P \in C$, and a curve of type II if $C \backslash L \cong \mathbf{A}^{1}$ for some line $L[\mathbf{3}]$. Let $B$ and $G$ be the groups of birational transformations of $\mathbf{P}^{2}$ (i.e., Cremona transformations) and automorphisms of $\mathbf{A}^{2}$, respectively. Identifying $\mathbf{P}^{2}-L$ with $\mathbf{A}^{2}$, where $L$ is a line, we regard $G$ as a subgroup of $B$ hereafter. Let $F(f)$ denote the set of fundamental points of $f \in B$. Then let us call $L$ a general line if $L \cap F(f)=\emptyset$. Two elements $f_{1}$ and $f_{2}$ of $B$ are said to be equivalent if there is a projective transformation $p$ satisfying $f_{2}=p f_{1}$. If $f$ is equivalent to an element of $G$, then $f(L)$ is a curve of type II for any general line $L$. On the other hand, in case $C \backslash L \cong \mathbf{A}^{1}$, there is an automorphism of $\mathbf{P}^{2}-L$ by which $C$ is carried to a line $[\mathbf{1}]$. Thus there is a close relation between automorphisms of $\mathbf{A}^{2}$ and curves of type II. We shall give a formulation of this fact in Remark 1 . Here we present the condition on which a Cremona transformation induces an automorphism of $\mathbf{A}^{2}$.

THEOREM. Let $f$ be a Cremona transformation. Suppose the exceptional curve of $f$ is irreducible and $f(L)$ is a curve of type II for a general line L. Then $f$ is equivalent to an element of $G$, hence $f\left(L^{\prime}\right)$ is also a curve of type II for every general line $L^{\prime}$.

Note that the theorem is not necessarily true in case we drop the condition of irreducibility (see Example 2). On the other hand, in case $f(L)$ is a curve of type I, $f\left(L^{\prime}\right)$ is not necessarily a curve of the type for another general line $L^{\prime}$ (see Example 3).

2. Proof of the Theorem. For a curve $C$ of type I, we define $R$ as in [3], i.e., let $\left(e_{1}, \ldots, e_{t}\right)$ be the sequence of the multiplicities of the infinitely near singular points of $P$ with degree $d \geq 3$. Then $R=R(C)$ is $d^{2}-\sum_{i=1}^{t} e_{i}^{2}-e_{t}+1$. We have shown that $R(C) \geq 2$ if $C$ is of type II [3]. Let $D$ and $D^{\prime}$ be the exceptional curves of $f$ and $f^{-1}$ respectively. Let $\sigma_{i}, i=1, \ldots, r$, be blow-ups such that $f \cdot \sigma$ is a morphism, where $\sigma=\sigma_{1} \cdots \sigma_{r}$. Since $D$ is irreducible, so is $D^{\prime}$. Hence the centers of the blow-ups are unique if the number $r$ is minimal. Thus $\sigma^{-1}[D]$ is first contracted by $f \cdot \sigma$, where [] denotes the proper transform. Note that $\sigma^{-1}[D]$ is a nonsingular rational curve with the self-intersection number -1 by Castelnuovo's

Received by the editors October 3, 1983.

1980 Mathematics Subject Classification. Primary 14 E07. 
criterion for contracting a curve [2]. Since $L$ is a general line, the transfrom $\sigma^{-1}(L)$ has self-intersection number 1 . The transforms $\sigma^{-1}(L)$ and $\sigma^{-1}[D]$ meet at only one point $Q$, since $f(L)$ is of type I. Therefore the intersection number of those curves at $Q$ is $e$, which is the degree of $D$. Suppose $e \geq 2$. Then, after the contraction of $\sigma^{-1}[D]$, the image of $L$ has a singular point with multiplicity $e$. Hence the degree of $f(L)$ is at least 3. From the above consideration we infer that $R(f(L))=2-e$. This means $f(L)$ is not of type II. Thus we have a contradiction, hence $D$ must be a line. Since $f$ induces an isomorphism between $\mathbf{P}^{2}-D$ and $\mathbf{P}^{2}-D^{\prime}$, the curve $D^{\prime}$ is also a line. Hence $f$ is equivalent to an element of $G$.

3. Let $(X, Y, Z)$ be a set of homogeneous coordinates on $\mathbf{P}^{2}$, and let $X, Y$ and $Z$ denote the lines defined by $X=0, Y=0$ and $Z=0$, respectively. Moreover, let $P=(1,0,0)$.

REMARK 1. Let $S$ be the set consisting of curves $C$ such that $C \cap Z=\{P\}$ and $C-\{P\} \cong \mathbf{A}^{1}$. Identifying $\mathbf{P}^{2}-Z$ with $\mathbf{A}^{2}$, we put

$$
G_{1}=\left\{f \in G \mid F\left(f^{-1}\right)=\{P\} \text { or } f(P)=P \text { according to } F(f) \neq \emptyset \text { or } F(f)=\emptyset\right\}
$$

and

$$
G_{2}=\{f \in G \mid f[Y]=Y\} .
$$

If $f \in G_{2}$ and $F(f) \neq \emptyset$, then $F\left(f^{-1}\right)=\{P\}$, otherwise the degree of $f^{-1}[Y]$ will be greater than 1 . Thus we see that $G_{2} \subset G_{1}$ and $G_{2}$ becomes a group; hence we consider the set $G_{1} / G_{2}$ of equivalence classes of $G_{1}$ with respect to $G_{2}$. Then there is a bijection between $G_{1} / G_{2}$ and $S$. In fact, let an element $f \in G_{1}$ correspond to $f[Y]$, which belongs to $S$. Thanks to [1] this mapping is surjective, hence defines the bijection. Thus, we may say that nonsingular rational curves in $\mathbf{A}^{2}$ passing through the infinite point $P$ can be "parametrized" by $G_{1} / G_{2}$.

EXAMPLE 2. Let $g$ be a Cremona transformation defined by $g(X, Y, Z)=$ $\left(g_{1}, g_{2}, g_{3}\right)$, where $n \geq 2$ and

$$
g_{1}=X Y^{n-1} Z+Z^{n+1}, \quad g_{2}=Y^{n+1}, \quad g_{3}=Y^{n} Z .
$$

Then $F(g)=\{P\}$ and the exceptional curves of $g$ are $Y$ and $Z$, but lines not passing through $P$ are carried to curves of type II.

EXAMPLE 3 . Let $\Delta$ be the curve defined by $X Z^{n-1}=Y^{n}$, where $n \geq 3$, and $g$ a nonlinear automorphism of $\mathbf{P}^{2}-\Delta$. Then $F(g)=\{P\}$ and $g(X)$ is of type I, whereas $f(L)$ is not of type I for any other general line $L$. For details see $[3]$.

\section{REFERENCES}

1. S. S. Abhyankar and T. T. Moh, Embeddings of the line in the plane, J. Reine Angew. Math. 276 (1975), 148-166.

2. R. Hartshorne, Algebraic geometry, Graduate Texts in Math., vol. 52, Springer-Verlag, 1977.

3. H. Yoshihara, Rational curve with one cusp, Proc. Amer. Math. Soc. 89 (1983), 24-26.

Department of Mathematics, Faculty of General Education, Nigata UniVERSITY, 950-21 NIIGATA, JAPAN 